\title{
HIV/AIDS Research Conducted in the Developing World and Sponsored by the Developed World: Reporting of Research Ethics Committee Review in Two Countries
}

\author{
Lisa Judy Chin, \\ Columbia University \\ Hoda Rifai-Bashjawish, \\ Columbia University \\ Kelly Kleinert, \\ Columbia University \\ Alexandra Saltman, \\ University of Toronto Medical School \\ Cheng-Shiun Leu, and \\ Columbia University \\ Robert Klitzman \\ Columbia University
}

\section{Abstract}

\begin{abstract}
We explored how often journal articles reporting HIV research sponsored by a developed country, but conducted in a developing country, mention research ethics committee (REC) approval from both countries, and what factors are involved. Of all such 2007 articles on Medline conducted in one of four developing countries $(\mathrm{N}=154)$, only $52 \%$ mentioned such dual approval. Mention of dual vs. single approval was more likely among articles with $\geq 50 \%$ sponsor country authors, and the United States as the sponsor country. Also, dual approval was more likely among articles that mentioned informed consent and funding, had $250 \%$ sponsor country authors, were biomedical (vs. psychosocial), and appeared in journals adopting International Committee Medical Journal Editors (ICMJE) guidelines. Dual approval was thus obtained in only half of the articles and was associated with ethical and logistic issues, indicating the need for clearer and more universally accepted guidelines.
\end{abstract}

\section{Keywords}

developing world; HIV/AIDS; policy; medical journals; research integrity; risks; benefits

\begin{abstract}
Research that is sponsored by the developed world and conducted in the developing world is often reviewed by a research ethics committee (REC) or Institutional Review Board (IRB) in each country, but such dual review poses numerous critical questions, and has received little, if any, scholarly attention. Importantly, how often and under what circumstances such dual review occurs and/or is reported is unknown.
\end{abstract}


The amount of medical research carried out in developing countries continues to expand. In 2005 , more than $20 \%$ of all clinical investigations submitted to the U.S. Food \& Drug Administration (FDA) took place outside of the U.S. and Western Europe (Tufts Center for the Study of Drug Development, 2007). In 2006, 41\% of active FDA-regulated principal investigators (PIs) were based outside the U.S. (Getz, 2007), and in 2007, 56\% of industryfunded phase III clinical trials listed on clinicaltrials.gov were based outside the U.S. (Glickman et al., 2009). This may reflect global health needs, and/or allow investigators to obtain larger numbers of participants for studies while lowering costs (Schmit, 2005).

Despite this increase in research in the developing world, however, the reporting of ethical protections in these situations in published reports of these studies is only beginning to receive attention (Emanuel et al., 2004; Kass, Dawson, \& Loyo-Berrios, 2003; Shapiro \& Meslin, 2001). In particular, while documentation of REC approval (note that we use the term "REC" below to refer to both RECs and IRBs) is seen as the "final check in ensuring the highest scientific and ethical standards, and a necessary step in protecting research subjects and maintaining public trust" (Myles \& Tan, 2003, p. 1212), only a few investigations have examined the practice of ethics review for research conducted in nonU.S. developed countries (Kjellström \& Fridlund, 2010), and in the developing world (Bavdekar, Gogtay, \& Wagh, 2008; Myles \& Tan, 2003; Sumathipala et al., 2008; Abdur Rab et al., 2008). Little research has compared RECs between different countries (Klitzman, 2007).

At times, dual approval may be obtained in such collaborative research (Emanuel et al., 2004; Kass et al., 2003; Shapiro \& Meslin, 2001; Varmus \& Schacter, 1997), and can be important, since approval by both a host country REC (which presumably knows the local conditions and context of the research setting better than the sponsor country REC), and a sponsor country REC (which may possibly be more familiar with the sponsor country investigators and REC regulations), can together help assure that the protocol has been reviewed as thoroughly as possible. In the United States, human subject research funded by the federal government is subject to 45 CFR 46, which states,

When research covered by this policy takes place in foreign countries, procedures normally followed in the foreign countries to protect human subjects may differ from those set forth in this policy.... In these circumstances, if a department or agency head determines that the procedures prescribed by the institution afford protections that are at least equivalent to those provided in this policy, the department or agency head may approve the substitution of the foreign procedures in lieu of the procedural requirements provided in this policy. (Department of Health and Human Services, 2005)

Presumably, this statement could thus be interpreted as mandating a review by an REC or its equivalent. But these regulations leave many questions unaddressed-e.g., concerning research that is not funded by the U.S. government. It is also unclear if foreign REC review is required, or if an agency head can just determine that the foreign researchers appear, on their own, to be following equivalent guidelines. Other countries' guidelines vary. Guidelines of the European Union (European Commission Group on Ethics in Science and New Technologies, 2003), Canada (Interagency Advisory Panel on Research Ethics, 2005), Japan (Ministry of Health, Labor, and Welfare, 2003), New Zealand (Health Research Council Ethics Committee, 2005), and the UK (Medical Research Council, 2004) require dual review. The national guidelines in Uganda recommend it (Uganda National Council on Science and Technology, 2007), and those in India (Indian Council of Medical Research, 2006) do not specifically mention it at all. Nigerian and Thai guidelines allow local RECs to accept another institution's review (National Health Research Ethics Committee, 2007; Medical Council of Thailand, 2007). Australian guidelines require the researcher to inform 
the Australian REC of the host country's ethics review process and capacity (National Health and Medical Council, Australian Health Ethics Committee, 2007).

We have found no studies that examine how often and when such dual approval in fact occurs. Given what little is known about how and when dual review occurs, an important first step is to assess how often published journal articles reporting on such research in fact mention dual REC approval.

In 1997, the International Committee of Medical Journal Editors (ICMJE) revised the uniform requirements for REC approval reporting in medical journals, and specified that authors should indicate whether research procedures followed the ethical standards of the appropriate institutional or national committee on human experimentation (International Committee of Medical Journal Editors [ICMJE], 1997). Nearly 800 journals are included on an online list of journals that claim to follow the ICMJE's uniform requirements for manuscripts, including many which publish articles on research carried out in developing countries (ICMJE, 2009). In response to ICMJE recommendations, the rates of reporting REC approval did seem to improve initially, as illustrated by a study of 103 Englishlanguage journals included in the Abridged Index Medicus, showing that compliance with REC approval requirements jumped from $45 \%$ to $76 \%$ between 1995 and 2005, and was associated with higher impact factors of journals (Rowan-Legg et al., 2009). But, at least in anesthesiology, compliance rates may vary based on type of study (e.g., clinical trial vs. observational study), and the specific journal (Matot, Pizov, \& Sprung, 1998; Myles \& Tan, 2003).

Moreover, studies of articles from the developing world suggest less compliance with these guidelines than that found in analyses of articles in the developed world. A 2008 study found that of Sri Lankan research articles, only 38\% documented REC/REC approval (Sumathipala et al., 2008), and of research papers published in two Indian pediatrics journals, only approximately 30\% reported REC/REC approval (Bavdekar, Gogtay, \& Wagh, 2008). Yet, the first of these studies did not explore other factors potentially associated with reporting of REC approval, such as author affiliations, type of research, or type of journal; and the second study examined only two journals from the same country, and failed to distinguish between Indian primary investigators and those not from India. Importantly, neither study examined the rates of reporting of dual REC approval, when research was sponsored by one country, but carried out in another.

Thus, we decided to explore how often dual REC approval is mentioned in journal articles reporting on research conducted in one of four developing world countries, with involvement of a developed world nation. We also examined what characteristics of the authors, journal, and study might be associated with differences in reporting of this approval. We chose to focus on HIV, since its effects are widespread (the pandemic affects approximately 38.6 million people worldwide), and is a crucial area of medical research in both developed and developing countries (Joint United Nations Programme on HIV/AIDS, 2006). HIV research also involves vulnerable and stigmatized populations-making protections of subjects particularly important —and has been the subject of much controversy (Angell, 1997; Angell, 2000; Lurie \& Wolfe, 1997). Of articles reporting on HIV research in the developing world, we have found that $80 \%$ did not mention conflicts of interest, and 32\% did not mention any REC approval (Klitzman et al., 2011; Klitzman et al., 2010). But for those studies that involve both a developed as well as a developing country, extremely crucial, separate questions arise that have never been explored-e.g., whether these studies mention dual review, and if so, how often, which do or do not, what they say, and what factors are involved. Hence, we have investigated these vital questions here. 


\section{Methods}

We conducted a search of the Medline database for all articles published in 2007 which met the following search criteria: (1) HIV research involving human subjects and (2) the research was sponsored by a developed country, but carried out in one of four developing countries (India, Thailand, Nigeria, or Uganda). We chose these countries since they have among the highest HIV prevalence in their respective geographic regions and the largest numbers of U.S.-sponsored HIV clinical trials (U.S. National Institutes of Health, 2008). We limited the search to papers with human subjects written in English. From the search, we included all articles that were available online through our university medical library, which holds 117,264 serials (Columbia University, 2008). We included only original research articles of studies involving human subjects, and excluded review articles, meta-analyses, letters, communications, brief reports, case reports, retrospective chart reviews, news articles, and data obtained from public databases (i.e., data collected for purposes other than research, such as state records and national epidemiological surveillance programs). Since this research did not involve human subjects (i.e., we did not interact with any subjects in any way), it was exempt from REC review at our institution.

Two research assistants (RAs), closely supervised by the senior author, independently coded the articles meeting the inclusion criteria. First, they each independently coded a sample of ten articles consisting of at least two articles from each country. They then developed a coding manual, and subsequently coded ten more articles independently and compared the results, revising the manual as necessary. They coded additional sets of ten articles independently, comparing results and discussing any disagreements (e.g., whether a study was more than minimal risk) until they reached complete consensus. All articles were then recoded using the final codebook. The articles were coded for factors in categories related to ethical characteristics (i.e., mention of informed consent, financial and nonfinancial compensation, and conflict of interest); study characteristics (i.e., type of intervention; the study being a clinical trial or not; the study being more than minimal risk or not); study population (i.e., whether the study involved vulnerable populations [e.g., infants or children, or individuals with HIV or other medical disorders]); funding characteristics (i.e., whether the funding source was mentioned, and whether the funding was from industry or not); authorship characteristics (percent of sponsor authors $<50 \%$ or $\geq 50 \%$; country of corresponding author; and name of sponsor country); and journal characteristics (i.e., location of the journal editors, and the journal being affiliated with ICMJE or not).

In this analysis, we chose mention of REC approval (i.e., dual REC approval, single REC approval, or no REC approval mention) as the primary outcome variable. To explore potential differences among countries, we assessed rates both among all countries in a fourway analysis (to see whether, overall, reporting of REC approval varied across countries), and between countries in two-way analyses (individual country vs. all other countries to see whether any particular country varied from the others). We used chi-square for these analyses because one cell (the number of studies from Nigeria that reported single REC approval) was zero, and we were thus unable to use polytomous regressions, as described below. To evaluate the associations between article characteristics and mention of REC approval, we employed both a simple and a multiple polytomous logistic regression analysis in order to obtain both p-values and odd ratios, indicating the direction of differences that emerged. Article characteristics that were found associated with mention of REC approval, with a p-value less than .10 from the simple polytomous regression analyses, were entered into the multiple polytomous regression model to determine the final predicting model. We report the odds ratio for the simple polytomous logistic regression analyses, and the adjusted odds ratio for the multiple polytomous logistic regression analyses and the corresponding pvalues and $95 \%$ confidence intervals $(\mathrm{CI})$. 


\section{Results}

Of the 590 articles found on Medline searches, 154 (26.1\%) met inclusion criteria (i.e., were sponsored by one country and conducted in another country). Of these 154 articles, approximately $52 \%(\mathrm{n}=80)$ reported REC approval from both host and sponsor countries, $22 \%(n=34)$ reported approval from only one REC, and 26\% $(n=40)$ mentioned no REC approval. Of the 34 reporting approval from only one REC, 27 mentioned an REC from the host country, 4 from the sponsor country, and 3 were unknown (i.e., REC approval was obtained, but the location of the REC was not mentioned).

As shown in Table 1, overall reporting of dual REC approval did not vary significantly by country. However, articles from Uganda were more likely, and those from India were less likely, than those from other countries to mention dual REC approval vs. no mention of REC approval. We also found that there was a trend for articles from Nigeria to be more likely than articles from other countries to mention dual REC vs. single REC approval.

Table 2 illustrates the associations between type of REC approval (dual, single, or no mention), and independent variables that reflect ethical and characteristics of the articles, using a simple polytomous logistic regression analysis.

The simple polytomous logistic regression analysis showed that articles that mentioned dual REC approval were more likely than those that mentioned only single REC approval to have $\geq 50 \%$ sponsor country authors $(\mathrm{OR}=5.182,95 \% \mathrm{CI}=2.179,12.324, \mathrm{p}=<0.001)$; to have the U.S. as the sponsor country $(\mathrm{OR}=2.535,95 \% \mathrm{CI}=1.083,5.933, \mathrm{p}=.032)$; and to have the corresponding author from a sponsor country $(\mathrm{OR}=5.46,95 \% \mathrm{CI}=2.13,14.08, \mathrm{p}<$ 0.001). Articles that reported dual REC approval were more likely than those that did not mention any REC approval to also mention informed consent $(\mathrm{OR}=13.148,95 \% \mathrm{CI}=$ $5.117,33,786, \mathrm{p}<0.001$ [i.e., conversely, those articles reporting informed consent were 13 times more likely to mention dual rather than no REC approval]); funding source (OR = $3.000,95 \% \mathrm{CI}=1.164,7.732, \mathrm{p}=.023)$; have $250 \%$ authors from sponsor country $(\mathrm{OR}=$ $2.739,95 \% \mathrm{CI}=1.247,6.018, \mathrm{p}=.012)$; appear in journals that had adopted ICMJE guidelines $(\mathrm{OR}=7.667,95 \% \mathrm{CI}=1.707,34.431, \mathrm{p}=.008)$; and involve biomedical (vs. psychosocial) research $(\mathrm{OR}=3.65,95 \% \mathrm{CI}=1.64,8.13, \mathrm{p}=.001)$.

We then simultaneously entered all the variables that were found above to have associations that were significant or trends into a multiple polytomous regression analysis. As shown in Table 3, findings suggest that articles that mentioned dual REC were also more likely than those that mentioned single REC approval to have $\geq 50 \%$ sponsor country authors (Adj. OR $=4.152,95 \% \mathrm{CI}=1.424,12.109, \mathrm{p}=.009$ ), and the U.S. as the sponsor country (Adj. OR = $3.407,95 \% \mathrm{CI}=1.220,9.515, \mathrm{p}=.019)$, and to have a corresponding author from a sponsor country (Adj. OR $=3.41,95 \% \mathrm{CI}=1.05,11.11, \mathrm{p}=.042$ ). Articles that reported dual REC approval were more likely than those that mentioned no REC review to mention informed consent (Adj. OR $=17.865,95 \% \mathrm{CI}=5.628,56.612, \mathrm{p}<0.001$ ) and funding source (Adj. $\mathrm{OR}=6.447,95 \% \mathrm{CI}=1.814,22.921, \mathrm{p}=.004)$, to involve biomedical research (Adj. OR = $3.21,95 \% \mathrm{CI}=1.12,9.26, \mathrm{p}=.032)$, and to have been published in journals that adopted ICMJE guidelines (Adj. OR $=7.457,95 \% \mathrm{CI}=1.188,46.825, \mathrm{p}=.032$ ).

\section{Discussion}

These data indicate that of articles we examined reporting human subject research sponsored by a developed country, but carried out in the developing world, only about half reported REC/REC approval from both countries. Of the articles that did not mention dual REC approval (i.e., mentioned single or no REC approval), approximately $41 \%$ had a U.S. sponsor, $40 \%$ had a sponsor-country corresponding author, and 34\% had mostly sponsor- 
country authors. Studies mentioning dual REC approval were also more likely than studies that mentioned single or no REC approval to be biomedical (vs. psychosocial), have more authors from the sponsor country, and appear in journals affiliated with the ICMJE, and, as a trend, have U.S. sponsorship. Of note, significant differences did not arise regarding the type of study (clinical trial or not), degree of risk in the study, or use of vulnerable populations. Articles from Uganda were more likely, and those from India were less likely, to mention dual rather than no REC approval.

These data suggest that both ethical and logistical factors are associated with whether articles report dual REC approval or not. Dual approval was associated with mention of other ethical features of articles (e.g., informed consent, nonfinancial benefits to participants, and conflicts of interest). Hence, some authors may simply be more likely than others to report ethical characteristics of their research in general. Indeed, mention of informed consent was highly significant in the multiple polytomous regression model, controlling for other logistical aspects of the research, when comparing mention of dual vs. no REC approval. Thus, authors' attention to these ethical issues appears independent of other characteristics of these authors or their research (e.g., type of research, location of corresponding author, etc.).

Several of the factors associated with the mention of dual REC appeared to be more logistical, reflecting structural variables-e.g., different regulatory environments in which researchers worked. Specifically, mention of dual REC approval was associated with the location of the corresponding author, the percentage of sponsor-country authors (which may suggest other aspects of the type and extent of the involvement of the sponsoring country), and the sponsor country being the U.S. (vs. elsewhere). These three variables all appeared to be significant in our model, when controlling for each other, suggesting that they each contributed independently to the likelihood that an article will report dual REC approval. Future research can explore these phenomena more fully. For instance, the percent of sponsor authors may in part reflect the likelihood that at least one of these authors' institutions will require REC approval that may then get documented in the paper.

The type of research itself (biomedical vs. psychosocial) may reflect ethical and logistical issues, as well as other issues. Biomedical research may involve higher risks to participants - and thus lead to researchers having more funding and obtaining and reporting dual approval. This factor was highly significant in comparisons of mention of dual vs. no REC approval. Yet, level of risk did not appear to be significantly associated with mention of dual REC approval vs. single or no mention of REC approval. However, studies may entail other kinds of risk that were not described in the published paper, and for which we thus did not code. The relatively high rates of nonreporting of dual REC approval is disturbing because REC approval from both host and sponsor countries may increase the likelihood that a review has occurred that is thorough, and aware as possible of the local context to help avoid the potential of researchers adopting lower standards.

The reporting differences that emerge between countries highlight, in part, the regulations of individual countries. The sponsor countries other than the U.S. were those in the European Union, Japan, Canada, and Australia. Regulatory guidelines among both these hosts and these sponsor countries range widely concerning dual REC approval, from requiring it, to recommending it, to not mentioning it. Yet articles involving any one of these countries vary widely in whether they mention dual, single, or no REC approval. No government regulations mention reporting of REC approval in published articles.

The fact that articles from India are less likely than those from other countries to report dual rather than no REC approval may reflect in part the fact that India's guidelines do not 
specifically mention dual review. But published guidelines alone do not wholly predict findings. While guidelines in the U.S. and in several other developed countries (e.g., the EU and Canada) appear similar, studies sponsored by the U.S. were more likely than those sponsored by other developed countries to report dual rather than single REC approval and, as a trend, to report dual rather than no REC approval. Hence, other factors-e.g., whether, and to what degree, guidelines are monitored, enforced, and followed - can play critical roles. Future research can probe in further detail when and how guidelines in each country are in fact monitored, enforced, and followed.

Of note, the national guidelines outlined here appear to have been in place by 2007, though Japan required dual REC approval only in 2008, and Uganda and Australia revised their regulations slightly in 2007, making it difficult, if not impossible, to know what exact guidelines researchers knew and/or followed when they conducted, wrote, and published their articles. Again, the present data raise several critical issues that future research can examine in more detail concerning whether, to what degree, how, and why authors and editors follow specific guidelines or not. Future investigations can also focus on articles from each non-U.S. sponsoring country separately.

The finding that single (vs. dual) REC approval was associated with investigators from sponsor countries other than the U.S. is noteworthy and surprising, since anecdotally, not obtaining optimal REC approval is often assumed to be due to a deficiency in the developing (not the developed) country.

It is possible that articles that did not mention dual or single REC approval nonetheless obtained it. We did not contact the authors of these articles to determine whether they in fact did so, but future research could pursue this possibility, contact authors directly, interview them (e.g., about whether, how, and why they made decisions concerning obtaining and reporting REC approval), and examine protocols themselves.

Research may also be exempt from REC review-e.g., if it analyzes de-identified data without direct contact with research participants; however, we reviewed the 72 articles that reported single or no REC approval, and found one only study with an explicit reference that the data were de-identified. Moreover, none of these studies reported that they were exempt from REC review. Thus, exemption from review does not appear to account for the relatively low rates here of reporting REC approval.

This study has several potential limitations. We only examined journals available online. Therefore, not all journals may have been available. Yet, even among these available journals, we still found relatively high rates of failure to mention dual REC approval. The sample size was sufficiently large to reach significance for several analyses, but larger sample sizes can permit assessment of additional variables, and can be pursued in future research.

\section{Best Practices}

Authors from developed countries conducting research in developing countries should take particular care to seek dual review and to so state in the publication of the research, as it has special significance for understanding sensitivity to local cultures and norms.

\section{Research Agenda}

Subsequent studies can also contact RECs directly to assess how often, where, and why they require dual REC approval, and what problems or discrepancies may emerge between the two RECs. Additional research can also probe impediments that might exist to adhering to 
regulations in various countries, and the ways that differing regulations may account further for variations found here.

\section{Educational Implications}

Researchers, including senior and junior researchers and trainees, in both the developing and the developed world (i.e., when collaborating with researchers abroad) need to be educated concerning the importance of obtaining and reporting dual REC approval in various situations.

\section{Acknowledgments}

This study was supported by NIH grant 5R01HG04214, "Views and Approaches to Research Integrity Among IRBs."

\section{References}

Abdur Rab M, Afzal M, Abou-Zeid A, Silverman H. Ethical practices for health research in the Eastern Mediterranean region of the World Health Organization: A retrospective data analysis. PLoS ONE. 2008; 3(5):e2094. [PubMed: 18461183]

Angell M. The ethics of clinical research in the third world. New England Journal of Medicine. 1997; 337(12):847-849. [PubMed: 9295243]

Angell M. Investigators' responsibilities for human subjects in developing countries. New England Journal of Medicine. 2000; 342(13):967-969. [PubMed: 10738056]

Bavdekar SB, Gogtay NA, Wagh S. Reporting ethical processes in two Indian journals. Indian Journal of Medical Science. 2008; 62(4):134-140.

Columbia University Libraries. (n.d.). Welcome to Columbia University Libraries. Retrieved July 30 2008 from http://www.columbia.edu/cu/lweb/about/

Department of Health and Human Services, Office for Human Research Protections. 45 CFR 46, Subpart A, B, C, D, and E. Retrieved December 15, 2010 from http://www.hhs.gov/ohrp/policy/ index.html\#topics

Emanuel EJ, Wendler D, Killen J, Grady C. What makes clinical research in developing countries ethical? The benchmarks of ethical research. Journal of Infectious Diseases. 2004; 189(5):930-937. [PubMed: 14976611]

European Commission Group on Ethics in Science and New Technologies. Ethical aspects of clinical research in developing countries. 2003. Retrieved December 152010 from http://ec.europa.eu/ european_group_ethics/docs/avis17_en.pdf

Getz KA. Global clinical trials activity in the details. Applied Clinical Trials. 2007; 16(9):42-44.

Glickman SW, McHutchison JG, Peterson ED, Cairns CB, Harrington RA, Califf RM, et al. Ethical and scientific implications of the globalization of clinical research. New England Journal of Medicine. 2009; 360(8):816-823. [PubMed: 19228627]

Health Research Council Ethics Committee. Guidelines on ethics in health research. 2005. Retrieved December 152010 from http://www.hrc.govt.nz/root/Publications/

Ethics_Reports_andGuidelines.html

Indian Council of Medical Research. Ethical guidelines for biomedical research on human participants. 2006. Retrieved December 152010 from http://icmr.nic.in/ethical_guidelines.pdf

Interagency Advisory Panel on Research Ethics. Tri-Council policy statement: Ethical conduct for research involving humans. 2005. Retrieved December 152010 from http://www.pre.ethics.gc.ca/ eng/policy-politique/tcps-eptc/

International Committee of Medical Journal Editors (ICMJE). Uniform requirements for manuscripts submitted to biomedical journals: Writing and editing for biomedical publication. 2008. Retrieved December 12008 from http://www.icmje.org/urm_full.pdf

International Committee of Medical Journal Editors (ICMJE). Uniform requirements for manuscripts submitted to biomedical journals. 2009. Retrieved from http://www.icmje.org/index.html\#ethic 
Joint United Nations Programme on HIV/AIDS. Report on the global AIDS epidemic: Executive summary. 2006. Retrieved August 1, 2008 from http://ata.unaids.org/Globalreports/ Global_Report2006/ENGLISH/executivesummary.pdf

Kass N, Dawson L, Loyo-Berrios NI. Ethical oversight of research in developing countries. IRB: Ethics \& Human Research. 2003; 25(2):1-10. [PubMed: 12833901]

Kjellström S, Fridlund B. Status and trends of research ethics in Swedish nurses' dissertations. Nursing Ethics. 2010; 17(3):383-392. [PubMed: 20444779]

Klitzman R. Views of the process and content of ethical reviews of HIV vaccine trials among members of U.S. institutional review boards and South African research ethics committees. Developing World Bioethics. 2007; 8(3):207-218. [PubMed: 19046258]

Klitzman R, Chin LJ, Rifai-Bashjawish H, Kleinert K, Leu CS. Disclosures of funding sources and conflicts of interest in published HIV/AIDS research conducted in developing countries. Journal of Medical Ethics. 2010; 36:505-510. [PubMed: 20663770]

Klitzman R, Kleinert KJ, Rifai-Bashjawish H, Leu CS. The reporting of IRB review in journal articles presenting HIV research conducted in the developing world. Developing World Bioethics. 2011 In press.

Lurie P, Wolfe SM. Unethical trials of interventions to reduce perinatal transmission of the human immunodeficiency virus in developing countries. New England Journal of Medicine. 1997; 337(12):853-856. [PubMed: 9295246]

Matot I, Pizov R, Sprung CL. Evaluation of institutional review board review and informed consent in publications of human research in critical care medicine. Critical Care Medicine. 1998; 26(9): 1596-1602. [PubMed: 9751600]

Medical Council of Thailand. Ethical guidelines for research on human subject in Thailand. 2007. Retrieved December 152010 from http://www.tmc.or.th

Medical Research Council. Research involving human participants in developing societies. 2004. Retrieved December 152010 from http://www.mrc.ac.uk/PolicyGuidelines/ EthicsAndGovernance/index.htm

Ministry of Health, Labor, and Welfare. Ethical guidelines for clinical studies. 2003. Retrieved December 152010 from http://www.ncgm.go.jp/rinri/main/03english.htm

Myles PS, Tan N. Reporting of ethical approval and informed consent in clinical research published in leading anesthesia journals. Anesthesiology. 2003; 99(5):1209-1213. [PubMed: 14576560]

National Health and Medical Research Council, Australian Health Ethics Committee. National statement on ethical conduct in human research. 2007. Retrieved December 152010 from http:// www.nhmrc.gov.au/guidelines/ethics/human_research/index.htm

National Health Research Ethics Committee. National code of health research ethics. 2007. Retrieved December 152010 from http://www.nhrec.net/nhrec/NCHRE_10.pdf

Rowan-Legg A, Weijer C, Gao J, Fernandez C. A comparison of journal instructions regarding institutional review board approval and conflict-of-interest disclosure between 1995 and 2005 . Journal of Medical Ethics. 2009; 35(1):74-78. [PubMed: 19103950]

Schmit J. Costs, regulations move more drug tests outside USA. USA Today. 2005 May 16.:1B.

Shapiro HT, Meslin EM. Ethical issues in the design and conduct of clinical trials in developing countries. New England Journal of Medicine. 2001; 345(2):139-141. [PubMed: 11450665]

Sumathipala A, Siribaddana S, Hewege S, Lekamwattage M, Athukorale M, Siriwardhana C, et al. Ethics review committee approval and informed consent: An analysis of biomedical publications originating from Sri Lanka. BMC Medical Ethics. 2008; 9(3):1-7. [PubMed: 18218097]

Tufts Center for the Study of Drug Development (CSDD). Outlook report. 2007. Retrieved December 152010 from http://csdd.tufts.edu/InfoServices/OutlookPDFs/Outlook200.pdf

Uganda National Council on Science and Technology. National guidelines for research involving humans as research participants. 2007. Retrieved December 152010 from http://uncst.go.ug/site/ documents/rihp_guide.pdf

U.S. National Institutes of Health. Clinical trials background information. 2008. www.clinicaltrials.gov

Varmus HM, Schacter D. Ethical complexities of conducting research in developing countries. New England Journal of Medicine. 1997; 337:1003-1005. [PubMed: 9309109] 


\section{Biographies}

Lisa Judy Chin is a postdoctoral research fellow at the HIV Center for Clinical and Behavioral Studies at the New York State Psychiatric Institute and Columbia University Department of Psychiatry. She is currently conducting research on ethics and policy issues concerning HIV/AIDS and has an interest in research ethics. She conducted the data analysis and helped prepare the manuscript.

Hoda Rifai-Bishjawish is a graduate of the Columbia University Fu Foundation School of Engineering and Applied Science with a major in Biomedical Engineering. She is currently a Fulbright Scholar conducting bioethics research in Syria and will be attending Columbia University Law School. She was involved with the design and data collection of the study.

Kelly Kleinert is a medical student at the Columbia University College of Physicians and Surgeons. She was involved with the design and data collection of the study.

Alexandra Saltman is a medical student at the University of Toronto. She assisted in the data analysis of the study and manuscript preparation.

Cheng-Shiun Leu is Assistant Professor of Biostatistics at the Columbia University Mailman School of Public Health. He provided statistical support for the study.

Robert Klitzman is Professor of Clinical Psychiatry at the Columbia University Department of Psychiatry. He is also the Director of the Ethics and Policy Core of the HIV Center for Clinical and Behavioral Studies at the New York State Psychiatric Institute and Department of Psychiatry and Director of the Masters of Bioethics Program at Columbia University. He conceived of and directed the study. 


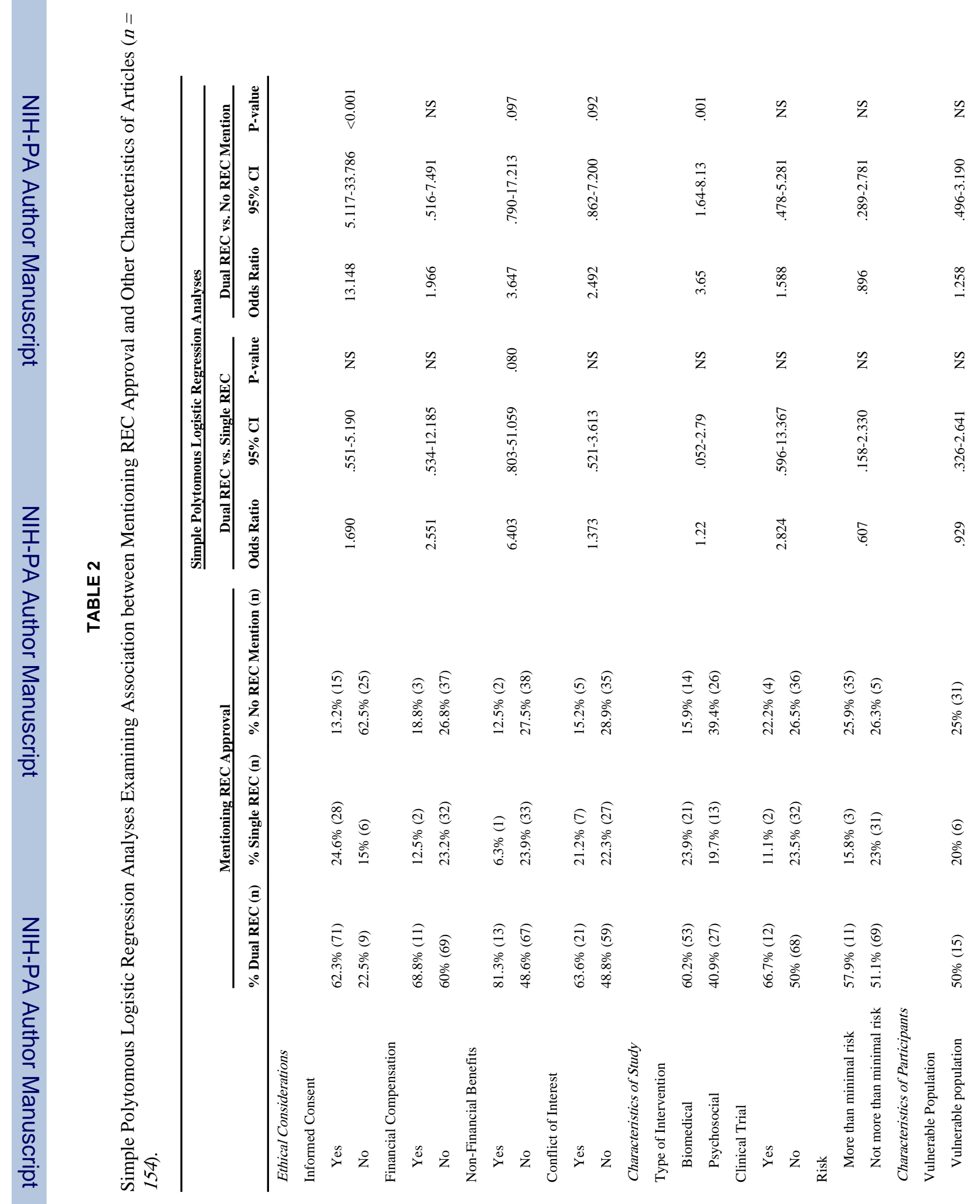




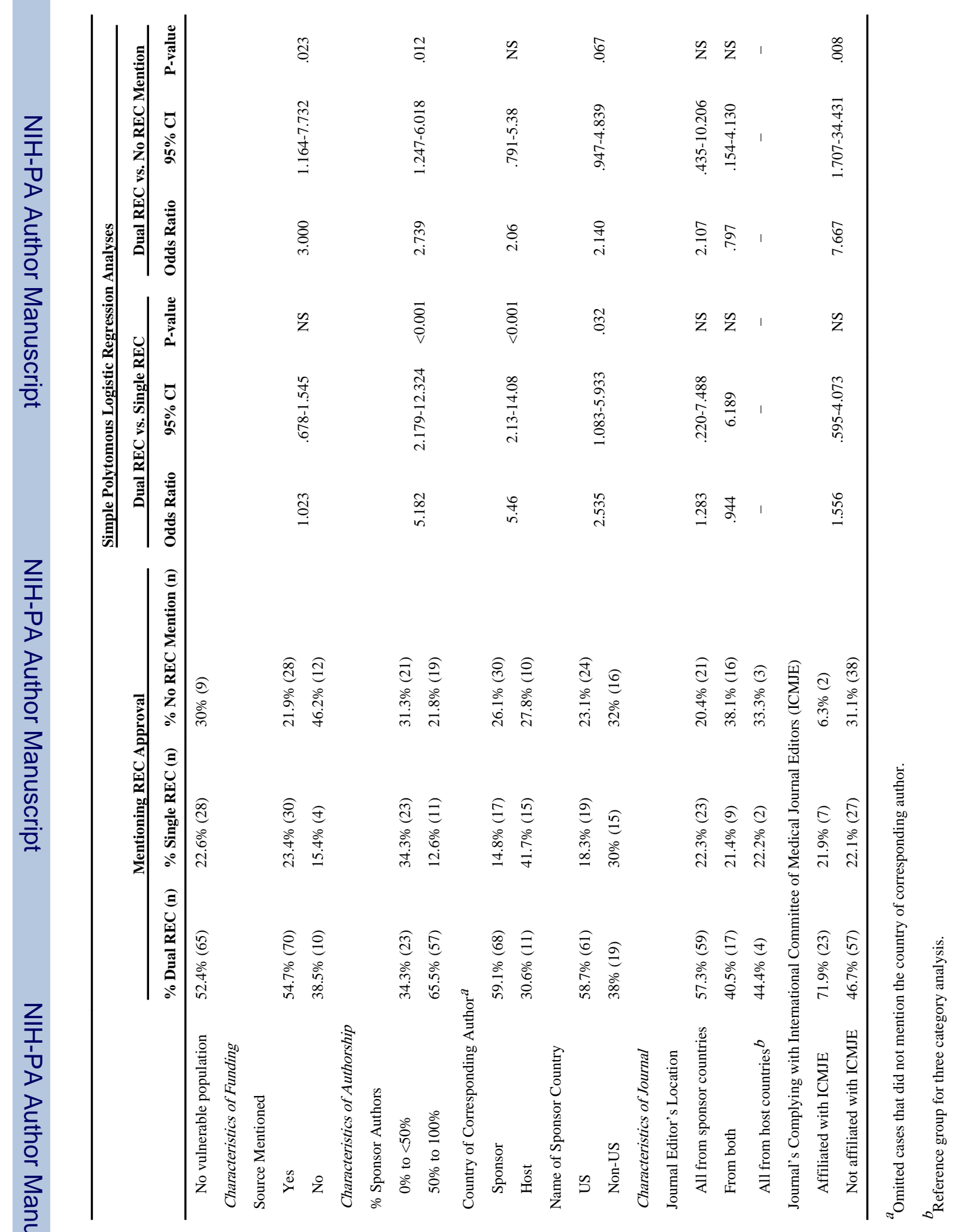


